\title{
D-LAJOA : DYNAMIC LOAD-AWARE JOINT OPTIMAL ALGORITHM IN MULTI-RADIO MULTI-CHANNEL WIRELESS MESH NETWORKS
}

\author{
Zhang Yong ${ }^{1}$, Liu $\mathrm{Han}^{2}$, Ma Wenjie ${ }^{3}$, Liu Kai-ming and Li Nan \\ ${ }^{1,2}$ State Grid Shandong Electric Power Corporation, Economic and Technical Research \\ Institute, Shandong, China \\ ${ }^{3}$ Beijing Guodiantong Network Technology Co.Ltd., Beijing, China
}

\begin{abstract}
To solve the problem that traffic network of rapid change is easy to fall into the situation of network congestion, based on the network load information, we use the average transmission time and local interface queue length information to find the path of the load balancing, and select minimum load channel through load-aware channel allocation. Considering interference suppression, load balancing, channel allocation and routing optimization design, we propose a dynamic load joint optimization algorithm DLAJOA, mainly designing channel load CL, adjustable weighting factor $\beta$ and the joint routing metrics. Simulations results demonstrate that D-LAJOA owns obvious advantages over average throughput, network overhead and packet loss compared with other algorithms.
\end{abstract}

\section{KEYWORDS}

Multi-channel Multi-radio, Wireless Mesh Networks, Load-aware, Joint design, Channel Assignment

\section{INTRODUCTION}

Multi-radio multi-channel Wireless Mesh Network (MRMC-WMN) is one of the most important technologies in the evolution of next-generation networks. Its multi-hop, self-organization, selfhealing and simple deployment is an effective way to solve the bottleneck problem of last mile. However, with the increase of the node density, it will cause the interference situation to deteriorate, the channel utilization rate is low and other rigid environment. Considering the interaction between channel allocation and routing, it needs joint channel allocation and routing to optimize the design to improve the channel utilization and network performance, and play the advantages of multi-radio multi-channel technology. Since it is a challenging interdependent task to find routing paths and allocate channels to links in the WMN, its focus is joint schemes about channel assignment and routing in multi-channel multi-radio wireless mesh networks.

MRMC-WMN network structure is similar to traditional WMN network structure, so its structure has three types: the client structure, infrastructure structure, and the hybrid structure [1].

\subsection{The Client Structure}

The client network structure, is also known as flat network structure. All client nodes have identical performance, and each client node and has a variety of functions. If the physical distance of two wireless nodes is over communication range, it can forward information through the relay node; if the physical distance of two wireless nodes is within the range of communication, it can

DOI: 10.14810/elelij.2017.6201 
directly exchange data. Due to its random mobility, the node energy consumption demand is higher, so the client node is adaptive to smaller structure for network scenario.

\subsection{The Infrastructure Structure}

The infrastructure structure is well known as backbone wireless Mesh network structure. Infrastructure structure can be divided into two layers, one layer is composed of fixed Mesh routers backbone layer, a layer is composed of terminal device access layer. That is to say, the infrastructure structure of Mesh nodes has the function of network access and flow together. As a result of the Mesh routers' fixed position in backbone layer, there is no limit to the energy consumption of nodes, which can provide a good network quality. Therefore it is suitable for larger network environment.

\subsection{The Hybrid Structure}

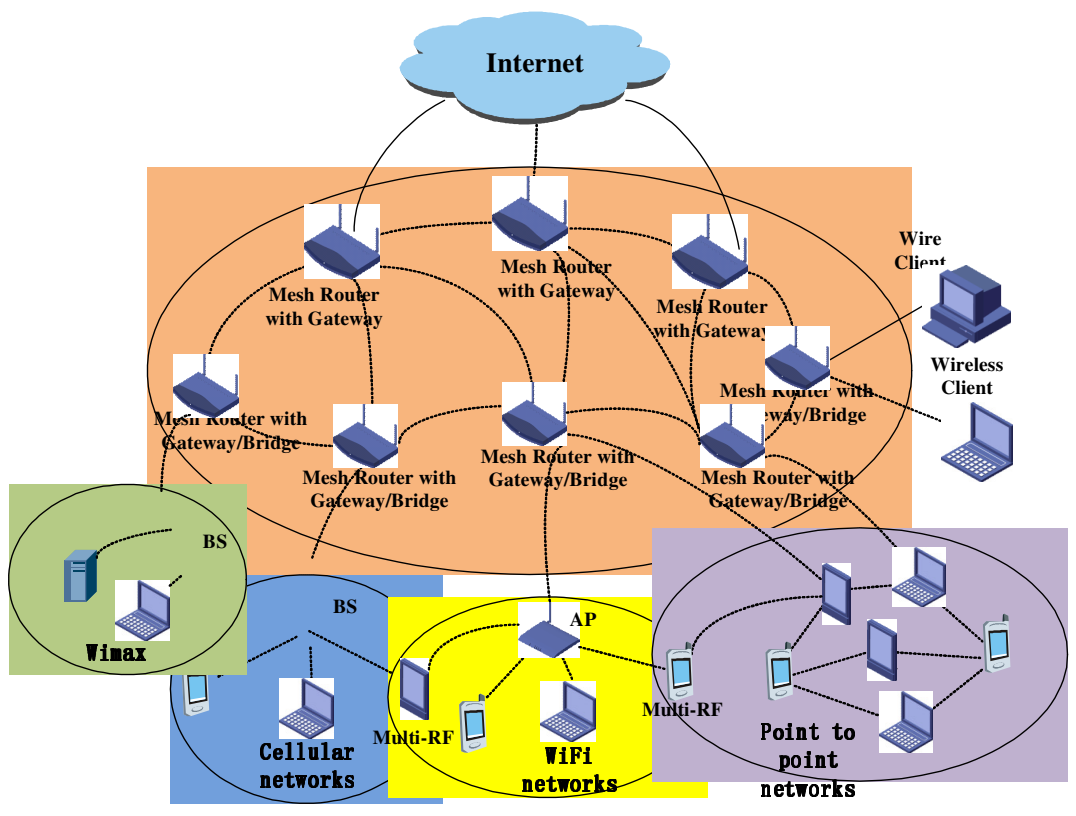

Figure 1. Hybrid structure in MRMC-WMN

As shown in figure below, hybrid type network structure is combination of the client network structure and backbone network structure. In hybrid network structure, the wireless Mesh nodes can be treated as both peer node and fixed Mesh router nodes, which can greatly expand the network coverage. The network structure is of good compatibility and maneuverability, so hybrid network structure is suitable for large-scale network environment with broad prospects.

Routing metrics is the key of the network construction. While in the study of traditional wireless Mesh network, a large number of studies have shown that the shortest path criterion is not the most optimal routing metric [2].Compared with the wired network, the distance of wireless network node is proportional to path fading, which means as the communication distance between nodes increases, path fading will gradually increase, so routing metric design with the minimum hop is obviously not comprehensive. Based on the conclusions above, the researchers build a new routing criterion [3-5] through parameters, such as the average transmission time, accumulative average transmission times network. The routing criterion design needs to fully consider the 
Electrical and Electronics Engineering: An International Journal (ELELIJ) Vol.6, No.2, May 2017

effect of channel allocation to network performance and network topology, For MRMC - WMN ,researchers have proposed many joint optimization algorithm, they can be mainly divided into the joint channel allocation, joint power control and joint load balancing algorithm [5-10].

In MRMC-WMN, an excellent strategy of channel allocation can assign appropriate channels for communication nodes to improve utilization rate of spectrum resources, plus efficient routing strategy can be quickly convergent and reduce the network delay. Therefore, it is very necessary and meaningful to realize routing joint optimization design in MRMC-WMN.

The paper further analyzes and compares the research status of joint optimal design of channel allocation and routing in multi-radio multi-channel wireless Mesh networks. In this paper, the necessity and feasibility of joint optimal design of channel allocation and routing in multi-radio multi-channel wireless Mesh networks are expounded in detail by referring to a lot of papers.

Based on the network load information, the paper finds the path of load balancing by using the average transmission time ETT and the local interface queue length information, and carries out the channel allocation through load sensing, and selects the load with low load channel. Based on the above work, this paper considers the interference suppression, load balancing, channel allocation and routing joint optimization design, mainly carries on the channel load CL, the adjustable weight factor $\beta$ and the joint route metric design, proposes a dynamic load sensing joint optimization algorithm D -LAJOA. D-LAJOA algorithm to load balancing and interference suppression as the optimization goal, in the process of finding the way to take into account the channel load conditions, and channel allocation, in order to achieve the effect of joint optimization. The simulation results show that the proposed algorithm D-LAJOA and D-WCETT, WCETT and other algorithms are compared and analyzed. The simulation results show that the proposed algorithm D-LAJOA has good performance in average throughput and average packet loss rate.

The paper is organized as follows. PART 1 introduces the basic concepts and infrastructure of multi-radio multi-channel wireless Mesh networks, and describes the design idea and key technologies of multi-radio multi-channel Mesh networks in detail. PART 2 gives regular system model and a exact description of some relative works about routing metrics. PART 3 proposes the Dynamic Load-Aware Joint Optimal Algorithm. PART 4 demonstrates that our simulation results outperforms other algorithms in terms of network throughput, network overhead and packet loss, and gives relevant analysis. PART 5 states final conclusion of this paper.

\section{SYSTEM MODEL /RELATIVE WORKS}

\subsection{Network Model}

We focus on the study of the multi-radio multi-channel wireless Mesh network, and MRMC WMN network topology can be expressed as a directed graph $G(N, L), N$ denotes the set of network nodes, $L$ denotes set of physical wireless links. $C_{k}=\{1,2,3\}$ denotes available channels set of multi-channel multi-radio wireless Mesh network. Wireless link $l_{u v}$ denotes the assigned radios from the sending node $u$ to the receiving node $v$, assigning available channel $\mathrm{k}$ for the link can be expressed as $l_{u v}^{k}$. In order to facilitate comprehension, the table below gives the key symbols meaning used in the paper. 
Electrical and Electronics Engineering: An International Journal (ELELIJ) Vol.6, No.2, May 2017

Table 1. Key symbols in MRMC WMN

\begin{tabular}{|l|l|l|l|}
\hline$C_{k}$ & Available channels set & $R_{t}$ & Node transmission range \\
\hline $\mathrm{L}$ & Physical wireless links set & $R_{u}$ & $\begin{array}{l}\text { Radio number configured for the } \\
\text { node } \mathrm{u}\end{array}$ \\
\hline $\mathrm{G}$ & Network directed graph & $C(u)$ & $\begin{array}{l}\text { Assigning available channels for } \\
\text { node } \mathrm{u}\end{array}$ \\
\hline$l_{u v}^{k}$ & $\begin{array}{l}\text { available channel } \mathrm{k} \text { for the link } \\
\text { between node u and node } \mathrm{v}\end{array}$ & $\mathrm{N}$ & mesh nodes set \\
\hline$R_{s}$ & Node carrier sense range & $R_{i}$ & node interference range \\
\hline
\end{tabular}

Because the number of radio for each wireless Mesh node configuration is limited, assigning channel number for the wireless Mesh nodes does not exceed the number of available radios wireless Mesh nodes, at the same time in order to improve the utilization rate of spectrum, different radios should be assigned with different channels, which means all of the radios of a wireless mesh node will not be assigned with the same channel. In addition, theoretically the radios of node interface can't be more than available orthogonal channels, there is a relationship as follows:

$$
|C(u)| \leq R_{u} \leq k, C(u) \subseteq C_{k}
$$

\subsection{Interference Model}

This paper adopts the Protocol interference Model which is commonly used. In MRMC - WMN, effective communication range of the wireless Mesh network nodes can be classified into three parts: node Transmission Range $R_{t}$, node Carrier Sensing Range $R_{s}$ and node Interference Range $R_{t}$. Transmission range $R_{t}$ denotes maximum range of mesh node successfully receiving signal without any interference, in general, the mesh node's transmission range is related to its transmission power, frequency. And node transmission power is proportional to the node transmission range; Carrier sensing range $R_{s}$ represents maximum range of nodes detecting other carrier signal sent by other nodes; node interference range $R_{i}$ denotes conflict range caused by nodes interference .In general, relationship is as follows:

$$
R_{t}<R_{i}=1.78 R_{t}<R_{s}=2.2 R_{t}
$$

If this paper needs to study load-aware situation of MRMC - WMN, we need to study its business flows model, there are two problems about business flows model in MRMC - WMN:

\section{The gateway hot issue}

Because most business flows are from MRMC-WMN gateway or to the gateway, it will cause the gateway hot issues, in addition it is so easy to lead to gateway load bottleneck problem, and it will seriously affect the network performance, so the paper will give full consideration to this feature about channel allocation and routing of the joint optimization design in MRMC-WMN.

\section{Link load imbalance problem}

In MRMC-WMN, the demand of each Mesh node is different, the link traffic load is generally different. Therefore, MRMC - WMN links traffic load are not balanced. The greater the link traffic, the greater the interference effect of the link around, so the paper in the channel allocation and routing optimization design fully consider the features. 


\subsection{Relative works}

There is a routing metric which can counts for Expected Transmission Count, called ETX[11].The node sends the probe packets for calculating Forward delivery rate and Reverse delivery rate while communicating with the neighbor nodes. Thus ETX consists of two parameter Forward delivery rate and Reverse delivery rate. [12] extends it into WCETT, Weighted Cumulative Expected Transmission Time, which acquires packet size, link bandwidth and ETX value to calculate the value of the ETT. Particularly, $X_{j}$ represents that a channel out of all available channels have the ETT of maximum value, to reflect the channels diversity. However, there are two limitations .one is that WCETT cannot adapt to dynamic network load, the other one is that WCETT did not solve the problem of inter-flow interference.

The concept of average queue length was introduced in the paper[13], and combine the WCETT as routing metric WCETT- LB, WCETT with Load Balancing. WCETT-LB algorithm using the average queue length LB to reflect the traffic load of the node, and dynamically choose the path with less load in the process of routing but the algorithm is applied to single radio single channel environment, in addition, its performance is only better than the minimum hop routing metrics.

D-WCETT routing metrics [14] can feed the need of being sensitive to dynamic mesh network load. D-WCETT considers the queue length of the link, and adjust dynamic weighting factor $\beta$ based on link level of load during routing. It is more suitable for more flows, especially more flows with different rates of multi-radio multi-channel wireless Mesh network .But it is incomplete using the Queue Length through interfaces as the link congestion level.

The key of channel allocation and routing joint optimization design is : network connectivity, network traffic and link-state information, technical limitations and the design of the routing metric [15].Plus, routing metric design considers some key factors: the path hop, channel interference, radio interface switch [16], computational complexity to reduce, convergence time decreasing and local constraints[17].

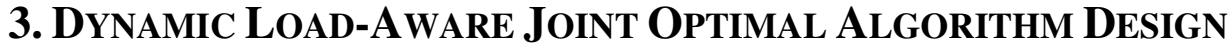

\subsection{Channel Load Design}

Expected Transmission Count, ETX, to collect forward delivery rate and reverse delivery rate of communication nodes by using the probe packets, the formula:

$$
\operatorname{ETX}=\frac{1}{d_{f} \cdot d_{r}}
$$

ETX denotes Expected Transmission Count, $d_{f}$ denotes forward delivery rate, $d_{r}$ denotes reverse delivery rate. Expected transmission time ETT, represent expected transmission time of sending a data packet successfully.

$$
E T T=E T X \cdot \frac{S}{B}=\frac{1}{d_{f} \cdot d_{r}} \cdot \frac{S}{B}
$$

$S$ represents data packet size, B denotes link bandwidth. Node with multi radios has the advantage of using multiple orthogonal available channels to maximize available bandwidth, we design channel load CL based on the average transmission time ETT and queue length, CL can be represented as: 
Electrical and Electronics Engineering: An International Journal (ELELIJ) Vol.6, No.2, May 2017

$$
C L(j)=\sum_{l \in c h a n n e l j} E T T_{l} \cdot Q_{l}
$$

$Q_{l}$ denotes the average buffer packets over link 1 between two nodes, $\mathrm{j}$ denotes channel index $\mathrm{j}$. Channel load CL not only can show the situation of the assigned channel load on the route, but also can reflect channel diversity. In short, we switch channels in the process of routing establishment according to channel load, choose the channel with minimum load, find the optimal routing, and achieve joint optimization.

\subsection{Adjustable weighting factor $\beta$}

To calculate network load we have used interface queue length (IFQ) which is a parameter indicating the total number of packets in the queue of a channel belonging to a node. The IFQ is a drop-tail buffer present at the MAC layer of IEEE 802.11 radios and contains outbound frames for the physical layer. A build up of frames indicates congestion due to high network traffic or low link quality. WMNs have a shared wireless medium which invites contention among nodes. This contention leads to congestion in the outbound IFQ of nodes. Queue lengths are properties of network nodes. Every network node possesses ingress and an egress queue in which incoming and outgoing packets are stored if the interface is unable to forward them immediately. The queue length gives an indication of the current state of the device: An empty queue is reflective of the fact that the device can process more traffic, whereas a full queue depicts a contented interface which cannot handle more packets. Usually, the queue length is small and as it becomes full, the node starts dropping packets.

In order to satisfy the interface function which can realize different data rate, the paper defines the Queue time (Queue Stay Interval,) before transmission data packet without need to stay in the interface Queue length IFQ, Queue time both can be expressed as:

$$
Q S I=\frac{I F Q}{B W}
$$

BW denotes the Bandwidth of channel. By normalizing the value it is ensured that the IFQ of different nodes with varying channel bandwidths are comparable.

$$
\beta=1-Q S I
$$

From (4), we know $0 \leq \beta \leq 1$. Whenever a route request is received at a node, the current value of link congestion on all available channels is obtained through the IFQ value. Links operating on different bandwidths are compensated to get QSI which is then normalized between 0 and 1, where 0 indicates an empty queue and 1 indicates a full queue. Depending upon the value of QSI, a value of $\beta$ is selected. In essence D-LAJOA gives higher weight to path length as compared to channel diversity when links are having higher load levels.

\subsection{Joint routing metrics}

From the part 3.1 and part 3.2 above, a joint routing metrics was proposed.

$$
D-L A J O A=(1-\beta) \mathrm{g} \sum_{j=1}^{k} C L(j)+\beta \mathrm{gmax}_{1 \leq j \leq k} C L(j)
$$

$\mathrm{K}$ denotes available channel number, $\mathrm{CL}(\mathrm{j})$ denotes network load of channel $\mathrm{j}, \quad \beta$ denotes adjustable dynamic weighting factor. When joint routing metrics shows the load of channel $\mathrm{j}$ is 
Electrical and Electronics Engineering: An International Journal (ELELIJ) Vol.6, No.2, May 2017

too large, it will automatically switch to the other available channels, to balance the network load and makes the algorithm own better performance in terms of throughput, network overhead, packet loss. Joint routing metric D-LAJOA, assign the available channel with interference suppression and the load balance to a certain link, by selecting the minimum $\max _{1 \leq j \leq k} C L(j)$ and according to the dynamic adjustable weighting factor beta, adapt to changable network traffic environment, which makes the optimal network throughput. Finally, based on the routing of assigning available channels to links, according to the joint routing metrics collections out of the calculation, select minimum routing metrics combination as routing and its assigned available channel.

This paper proposed to design the routing table for D- LAJOA routing metric. When D-LAJOA algorithm choose the next hop, routing table can get these parameters, like available channel, the weighting factor $\beta$ and channel load by the routing metric calculation.

Table 2. D-LAJOA routing table

\begin{tabular}{|l|l|l|l|l|l|}
\hline $\begin{array}{l}\text { Destination } \\
\text { node }\end{array}$ & $\begin{array}{l}\text { Next } \\
\text { hop }\end{array}$ & $\begin{array}{l}\text { Channel } \\
\text { In } \\
\text { use }\end{array}$ & $\begin{array}{l}\text { Channel } \\
\text { load }\end{array}$ & $\begin{array}{l}\text { adjustable } \\
\text { weighting } \\
\text { factor } \beta\end{array}$ & $\begin{array}{l}\text { D-LAJOA } \\
\text { routing } \\
\text { metrics }\end{array}$ \\
\hline & & & & & \\
\hline
\end{tabular}

The routing table items include: destination node address, and the next hop address, available channels to the next node, and the load status of the available channel, the adjustable weighting factor $\beta$ and the value of joint routing metric according to the formula (5).

\subsection{Joint routing metrics}

Step 1: initialization phase. Node assign available channels randomly in the network topology, but need to follow two principles below: (1) different radios of one node should be assigned with orthogonal channels; (2) the unbounded radios should be assigned with channels in priority.

Step 2: choose the path and assign channels. According to the network topology after channel assignment initialization, joint routing metric is used to set up a minimum load-balancing path for routing. According to the established route table, record all the assigned available channel and channel load CL of one hop path, we reassign available channel for node which is of maximum CL value.

Step 3: iteration. If the result of $\mathrm{N}+1$ th channel assignment is the same as the $\mathrm{Nth}$ channel assignment result, and then the channel and route is the best. Otherwise, jump to step 2;

Step 4: update phase. The last time result of the path selection and channel assignment can be taken as the final result.

\section{SIMULATION RESULTS}

The simulation parameters are shown in the table below: 
Electrical and Electronics Engineering: An International Journal (ELELIJ) Vol.6, No.2, May 2017

Table.3. Simulation parameter

\begin{tabular}{|l|l|}
\hline Simulation parameter & Value \\
\hline Simulator & NS2.35 \\
\hline Routing protocols & MRMC-AODV/JCEED/D-LAJOA \\
\hline Antenna & Omni-directional \\
\hline Mesh nodes number & $4 / 8 / 12 / 16 / 20 / 24 / 28 / 32 / 36 / 40$ \\
\hline Simulation area & $1000 \mathrm{~m} * 1000 \mathrm{~m}$ \\
\hline node radios number & 3 \\
\hline available channels number & 3 \\
\hline flow number & $2 / 4 / 6 / 8 / 10 / 12 / 14 / 16 / 18 / 20$ \\
\hline Packet transmission rate & $32 \mathrm{pkts} / \mathrm{sec}$ \\
\hline channel bandwidth & $11 \mathrm{Mbps}$ \\
\hline IFQ MAX data & $50 \mathrm{packets}$ \\
\hline packet loss rate & 0.01 \\
\hline transmission/interference range & $250 \mathrm{~m} / 550 \mathrm{~m}$ \\
\hline Working frequency & $2.4 \mathrm{GHz}$ \\
\hline Simulation time & $100 \mathrm{~s}$ \\
\hline
\end{tabular}

\subsection{Network throughput}

When the number of business flow gradually increases, the average throughput of D - LAJOA algorithm we proposed in this paper is on the rise along with other three comparative algorithms. The figure shows that D - LAJOA algorithm is of the best performance. Because MRMC AODV algorithm lacks tackling load balance, when it is in the network congestion situation. D WCETT and WCETT algorithm consider and realize network load balance, to a certain extent, but the optimization effect is limited. D - LAJOA algorithm fully considers the channel load and dynamic weighting factor in the process of routing, and realizes the joint optimization of channel allocation and routing select, so it can effectively improve the network throughput spontaneously.

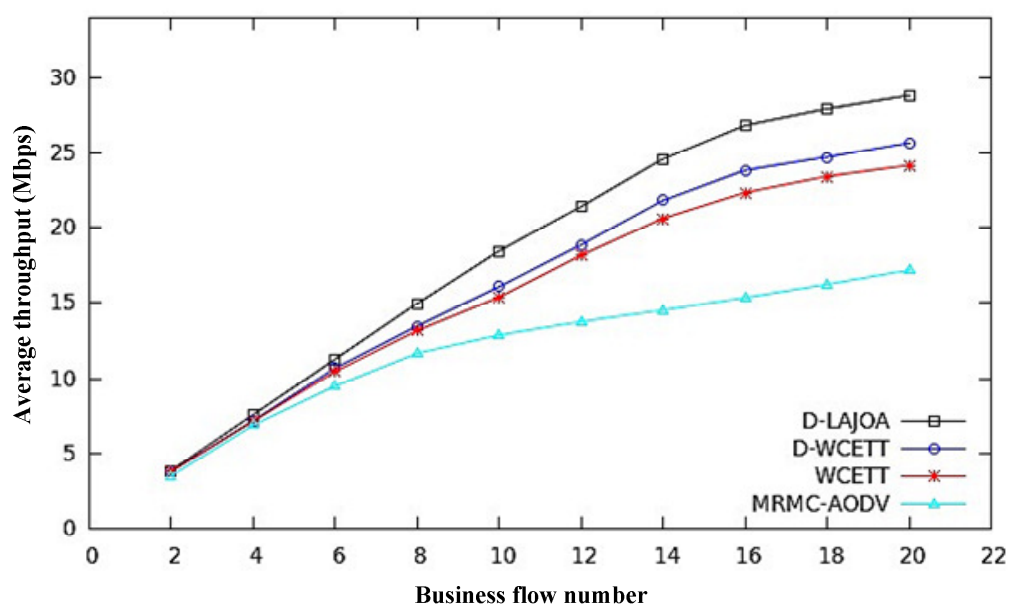

Figure 2. Comparison of average throughput performance in different data flow numbers

\subsection{Network Overhead}

Network overhead: the same as routing packet overhead, the ratio of control packets generated and number successfully received data packets .Network overhead rate in decreasing order: DWCETT> D-LAJOA > WCETT> MRMC-AODV. Because the D-LAJOA algorithm uses the local property Interface Queue Length as a factor of reflecting the network load, it will not cause 
Electrical and Electronics Engineering: An International Journal (ELELIJ) Vol.6, No.2, May 2017

additional network overhead. Compared to MRMC-AODV algorithm, the other three algorithms will increase the network overhead. But the algorithm D-LAJOA control network overhead in the joint design at the same time, we also can tell from the figure that the network overhead performance of D-LAJOA algorithm is better than that of D-WCETT algorithm.

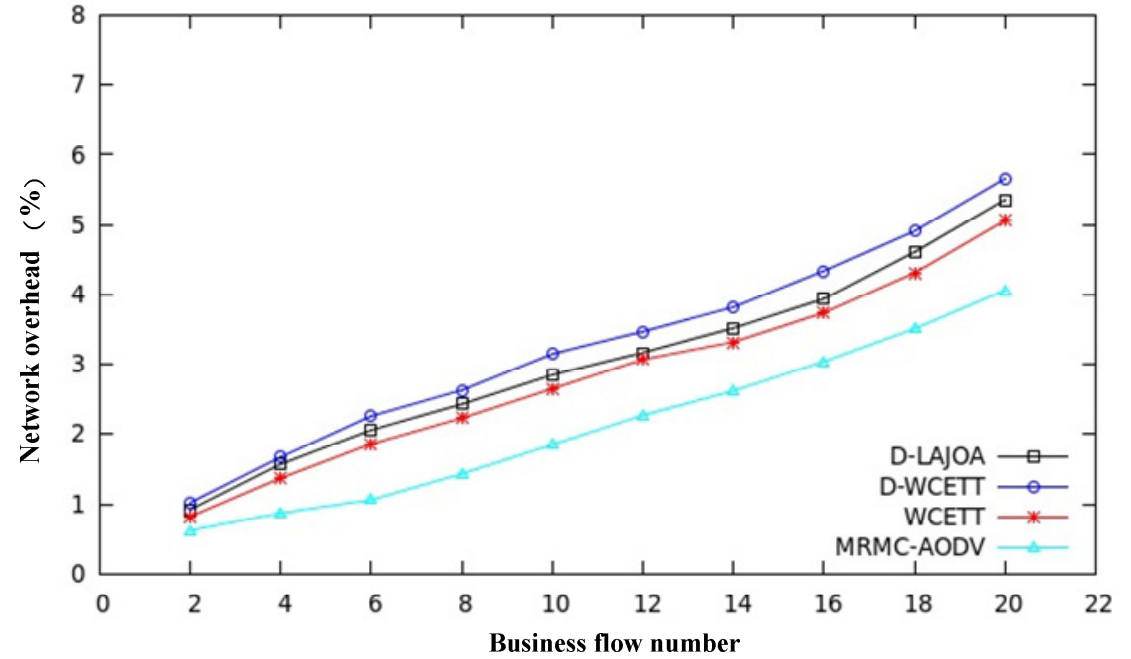

Figure 3. Comparison of network overhead performance in different data flow numbers

\subsection{Packet loss}

The figure above shows average packet loss in decreasing order: MRMC-AODV $>$ WCETT $>$ DWCETT $>$ D-LAJOA, D-LAJOA algorithm under the network congestion situation can effectively achieve load balance and interference suppression index, in turn, reduce the packet loss rate of the network.

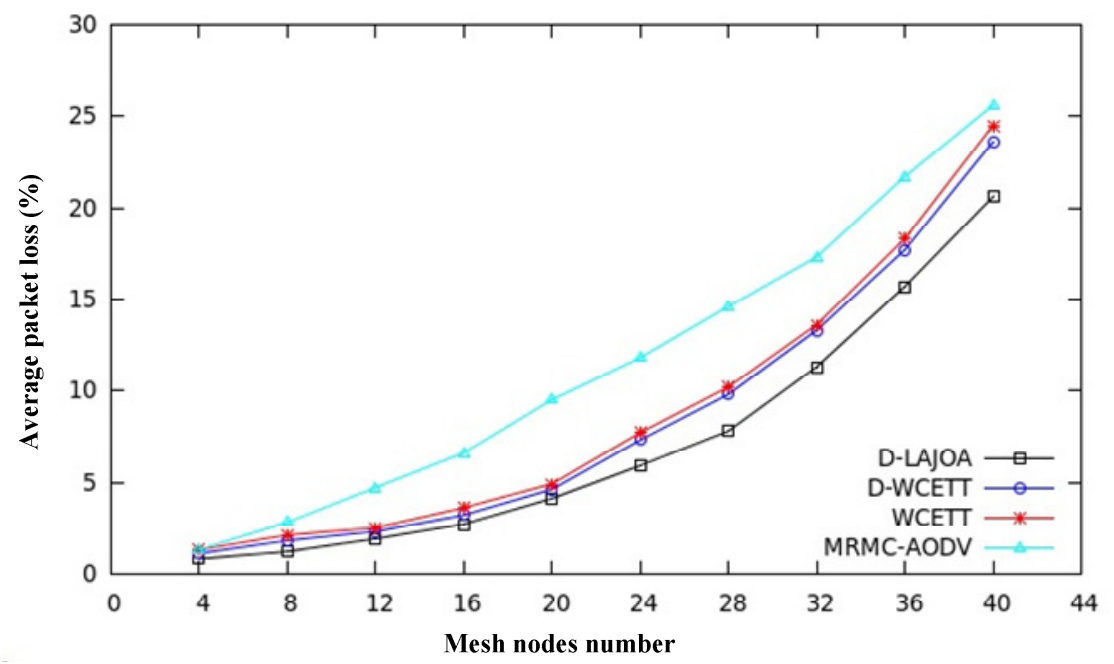

Figure 4. Comparison of packet loss ratio performance in different numbers of nodes

\section{CONCLuSion}

Considering interference suppression, load balancing, channel allocation and routing optimization design, we propose a dynamic load joint optimization algorithm D-LAJOA, targeting load balancing and interference suppression as the optimization goal and design channel load CL, 
Electrical and Electronics Engineering: An International Journal (ELELIJ) Vol.6, No.2, May 2017

adjustable weighting factor $\beta$ and the joint routing metrics. We can tell from simulation analysis comparison among D-LAJOA, D- WCETT and WCETT, that D-LAJOA owns obvious advantages over average throughput, network overhead and packet loss.

\section{REFERENCES}

[1] Y. Cheng, H. Li, D. M. Shila, and X. Cao. A systematic study of maximal scheduling algorithms in multiradio multichannel wireless networks[J]. IEEE/ACM Trans. Netw., vol. 23, no. 4, pp. 13421355, 2015.

[2] Skalli H, Ghosh S, Das S K, et al. Channel assignment strategies for multiradio wireless mesh networks: issues and solutions [J]. Communications Magazine, IEEE, 2007, 45(11): 86-95.

[3] H. Skalli, S. Ghosh, S. Das, L. Lenzini, and M. Conti, "Channel Assignment Strategies for Multiradio Wireless Mesh Networks:Issues and Solutions,” IEEE Comm. Magazine, vol. 45, no. 11,pp. 86-95, Nov. 2007.

[4] A. Subramanian, H. Gupta, S.R. Das, and J. Cao, "Minimum Interference Channel Assignment in Multi-Radio Wireless Mesh Networks," IEEE Trans. Mobile Computing, vol. 7, no. 12, pp. 14591473, Dec. 2008.

[5] M. Alicherry, R. Bhatia, and E. Li, "Joint Channel Assignment and Routing for Throughput Optimization in Multiradio WirelessMesh Networks," IEEE J. Selected Areas in Comm., vol. 24, no. 11,pp. 1960-1971, Nov. 2006.

[6] Ramachandran K N, Belding E M, Almeroth K C. Interference-aware channel assignment in multiradio wireless mesh networks[C]. IEEE Internation Conference on Computer Communications, 2006:1-12.

[7] Kaur N, Saini J.S.Performance enhancement of 802.11 based wireless mesh network by using MultiRadio Multi-Channel[J], IEEE ICGCE ,pp.71-76,2013.

[8] Mady Dzal G.I., Suili Feng and Weijun Tang.Joint fair resource allocation for multi-radio multichannel in wireless mesh networks: An efficient preemption approach[C].IEEE WSWAN,pp.16,March,2015.

[9] Gervais I Mady Dzal.; Suili Feng; Weijun Tang; Wei Feng; Yuan Liu.Joint Fair Resource Allocation for Multi-radio Multi-channel MeshNetworks with Flow Demand Constraint[C].CSNT, pp.233238,2014 .

[10] Xianghui cao, Lu Liu, Wenlong Shen and Yu Cheng. Distributed Scheduling and Delay-Aware Routing in Multi-Hop MR-MC Wireless Networks[J].IEEE Transactions on Vehicular Technology,2015.

[11] H. Li, Y, Cheng, C. Zhou, and W. Zhuang, Minimizing end-to-end delay: a novel routing metric for multi-radio wireless mesh networks.,IEEE INFOCOM, pp. 46-54, 2009.

[12] D. S. De Couto, D. Aguayo, J. Bicket, and R. Morris.A highthroughput path metric for multi-hop wireless routing[J].Wireless Networks, vol. 11, no. 4, pp. 419-434, 2005.

[13] R. Draves, J. Padhye, and B. Zill.Routing in multi-radio, multi-hop wireless mesh networks[C].Conf. on Mobile Computing and Networking, 2004, pp. 114-128.

[14] L. Ma and M. K. Denko, "A routing metric for load-balancing in wireless mesh networks," in Proc. of 21st Int. Conf. on Advanced Information Networking and Applications Workshops, vol. 2, 2007, pp.409-414. 
Electrical and Electronics Engineering: An International Journal (ELELIJ) Vol.6, No.2, May 2017

[15] R.F. Ali, A.K. Kiani and A.A. Pirzada.Load Dependent Dynamic Path Selection in Multi-Radio Hybrid Wireless Mesh Networks[C].IEEE Wireless Communications and Networking Conference WCNC, pp. 2044-2049, 2014.

[16] H. Li, Y, Cheng, C. Zhou, and W. Zhuang, Minimizing end-to-end delay: a novel routing metric for multi-radio wireless mesh networks., IEEE INFOCOM, pp. 46-54, 2009.

[17] H.Li,Y.Cheng,C.Zhou , and W.Zhuang.Routing metrics for minimizing end-to-end delay in multiradio multi-channel wireless networks[J].IEEE Trans.2007. 